\title{
El uso de los dispositivos electrónicos móviles como herramienta docente de una asignatura de Grado.
}

\section{$M^{a}$ Ángeles Alcaide ${ }^{a}$ y Elena De la Poza ${ }^{b}$}

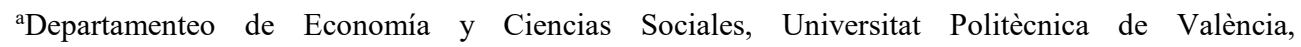
manalgon@upv.es, bDepartamenteo de Economía y Ciencias Sociales, Universitat Politècnica de València, elpopla@esp.upv.es.

\begin{abstract}
Currently, University students of Bachelor degrees are accustomed to constant use and consultation on the Internet and social networks through their laptops, tablets and mainly mobile phones "smartphones." That is why these tools can be an obstacle in student-teacher communication. On the other hand, it is possible to consider the use of them as teaching tools, integrating them in the classrooms to facilitate learning, as long as the student is aware and responsible for the use that makes them. Thus, the use of electronic devices could be catalysts of the teaching-learning process.

This contribution analyses the suitability of the use of electronic mobile phone devices in the classroom. For this, first, we analyze the habits and the use that the students make of these electronic devices. Secondly, the policy of the use of electronic devices in the in-class sessions of the subject "Business" taught in the degree of Aerospace Engineering in relation to the transversal competence N7, which is point of control, and finally, a SWOT analysis of the main aspects of its implementation in the teaching is carried out.
\end{abstract}

Keywords: Transversal competencies, ethical responsibility, professional responsibility, smartphone, social networks, ICT, electronic devices.

\section{Resumen}

En la actualidad, los alumnos que estudian titulaciones universitarias están acostumbrados al uso y consulta constante en Internet y en redes sociales a través de sus ordenadores portátiles, tablets y fundamentalmente teléfonos móviles "smartphones". Es por ello, que estas herramientas pueden ser un obstáculo en la comunicación alumno-docente. Por otra parte, es posible considerar la utilización de las mismas como herramientas docentes, integrándolas en las aulas para facilitar el aprendizaje, siempre y cuando el alumno sea consciente y responsable del uso que hace de ellas. Así, el uso de dispositicos electrónicos podrían ser catalizadores del proceso de enseñanza-aprendizaje.

Esta contribución analiza la idoneidad del uso de los dispositivos electrónicos de telefonía móvil en el aula. Para ello, en primer lugar, se analizan los hábitos y el uso que hacen los alumnos de estos dispositivos electrónicos. En segundo lugar, se detalla la política de utilización de 
dispositivos electrónicos en las sesiones de clase de la asignatura de Empresa impartida en el Grado de Ingeniería Aeroespacial en relación a la competencia transversal $N 7$, que es punto de control, y por último, se realiza un análisis DAFO de los principales aspectos de su implementación en la docencia.

Palabras clave: competencias transversales, responsabilidad ética, responsabilidad profesional, smartphone, redes sociales, TIC, dispositivos electrónicos.

\section{Introducción}

En los últimos años el uso de los dispositivos electrónicos en la docencia ha experimentado un progresivo crecimiento. Hasta hace poco más de diez años, el uso de estos dispositivos por parte de los alumnos de Educación Superior dentro del aula se limitaba a las sesiones de laboratorio donde se disponía de ordenadores fijos. Sin embargo, cada vez es más habitual que los alumnos traigan sus ordenadores portátiles y tablets a cualquiera de las sesiones de clase, y que estos sustituyan a las libretas y apuntes en formato papel. Por otra parte, el uso tan extendido de los smartphones, hace que hoy en día cualquier estudiante vaya acompañado del suyo tanto dentro como fuera del aula. Por todo esto, en estos últimos años se ha planteado la integración de estos dispositivos en la docencia; sin embargo, su uso dentro de las aulas conlleva tanto aspectos positivos como negativos. A pesar de ello, actualmente, ya es una realidad su implementación en muchas aulas de Educación Superior Universitaria. Varios estudios dan prueba de ello (Organista y Serrano, 2011; Arnáiz y Álvarez, 2016), y también analizan sus inconvenientes frente a los beneficios que pueden reportar al proceso de enseñanza-aprendizaje. Entre los principales inconvenientes, cabe destacar el uso inapropiado que pueden hacer los alumnos de estos dispositivos si se les permite utilizarlos en las aulas (Alonso et al, 2016). Por otra parte, si se implementan de manera adecuada son catalizadores de la enseñanza (Dominic, 2017). En cualquier caso son los docentes quienes deben liderar el proceso de integración de las TIC en el aula, ya que influye en la adquisición de las competencias desarrolladas en sus respectivas asignaturas (Brazuelo et al, 2017).

Concretamente, en la asignatura "Empresa" del Grado en Ingenieria Aeroespacial impartida en la Universitat Politècnica de València, es punto de control la Responsabilidad Ética, Medioambiental y Profesional. Esta competencia, según el proyecto de competencias transversales de la Universitat Politècnica de València (UPV, 2019) queda definida como el conjunto de conocimientos, habilidades, destrezas y actitudes útiles para interactuar con el entorno, de forma ética, responsable y sostenible, en orden a evitar o disminuir los efectos negativos producidos por las prácticas inadecuadas que ocasiona la actividad humana y para promover los beneficios que pueda generar la actividad profesional en el ámbito medioambiental, teniendo en cuenta sus implicaciones económicas y sociales. Así, la competencia transversal $\mathrm{N}^{\circ} 7$ aglutina tres ámbitos, que se detallan a continuación: 
- La responsabilidad ética, la cual se refiere a orientar la acción humana en un sentido racional, por lo que se relaciona con las acciones y su valor moral.

- La responsabilidad medioambiental es la imputabilidad de una valoración positiva o negativa por el impacto ecológico de una decisión y se refiere, generalmente, al daño causado a otras especies, a la naturaleza o a las futuras generaciones, por las acciones o las no-acciones de otro individuo o grupo.

- La responsabilidad profesional surge, en este punto, como incluida dentro de la responsabilidad moral, alcanzando el interior de nuestra conciencia y con dos objetivos primordiales: evitar toda falta voluntaria y disminuir, en lo posible, el número de faltas involuntarias por debilidad humana, flaqueza propia o negligencia ajena.

Esta competencia se trabaja en dos dimensiones según el esquema de la figura 1.

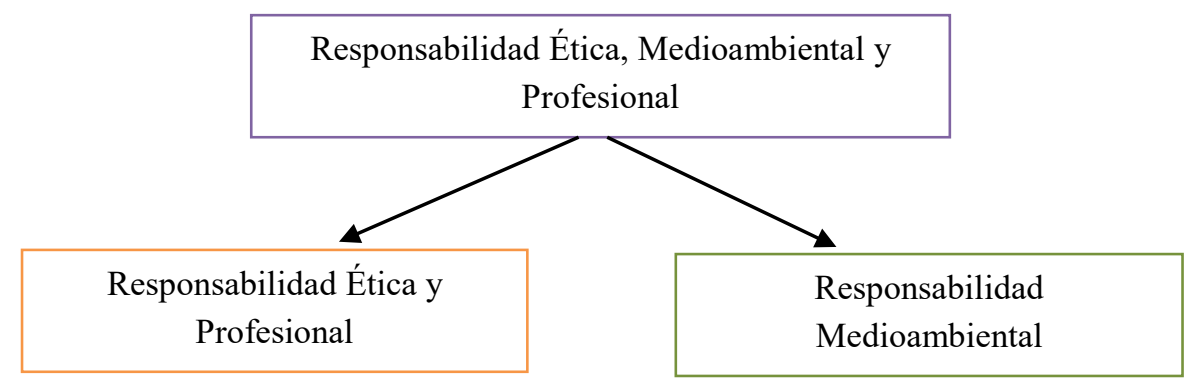

Fig. 1 Dimensiones de la Responsabilidad ética, medioambiental y profesional

Fuente: Elaboración propia según Competencias Transversales UPV (2019).

En esta comunicación nos centramos únicamente en la primera dimensión: la Responsabilidad Ética y Profesional.

La ética es necesaria en la Educación Superior Universitaria, ya que constituye el desarrollo de la personalidad y carácter de las personas, elementos que proporcionan criterios y juicio ético, que a su vez contribuyen a fortalecer las cualidades de los futuros profesionales (Ibarra, 2005). De igual modo, la responsabilidad profesional es la capacidad moral de cada persona, ya que cada uno debe desarrollar aquellos valores que le permitan ejercer su profesión dignamente y con integridad, es decir, con justicia, responsabilidad, discreción y honestidad (Hernández, 2010). Así pues, estos valores y capacidades enmarcados en la competencia transversal $\mathrm{N}^{\circ}$, contribuyen al desarrollo completo de los alumnos de educación superior para su integración en la sociedad y en la vida profesional.

Una manera de evaluar esta competencia en las aulas es a través de la observación.

En la asigantura "Empresa", analizada en esta contribución, el alumno es informado al inicio del curso de las normas y criterios a seguir en las sesiones de clase, y sobre el uso de dispositivos electrónicos durante las mismas. Así, es posible valorar el comportamiento ético y responsable del alumno en base al cumplimiento de las normas con respecto al uso de las Tecnologías de la Información y Comunicación (TIC); ya que, aunque por una parte la implementación de las TIC como herramientas didácticas para la construcción del conocimiento se impone en todos los niveles educativos (Navés, 2015), por otra parte, es 
necesario controlar su uso para no interferir en el proceso de enseñanza-aprendizaje, y en especial, con el actual alumnado de la llamada "Generación en red" o "Generation red" (Tapscott, 2009) o "Millenials" (Meso et al, 2011), que se ha acostumbrado a la interactividad que promueven las redes sociales, y al uso de teléfono móviles "Smart", e Internet. De hecho, las redes sociales se han convertido en esenciales en sus vidas, y los jóvenes las utilizan con frecuencia con fines diversos (Martí y García, 2018).

Concretamente, en la asignatura, el criterio seguido sobre el uso de estos dispositivos electrónicos es su prohibición en el aula como regla general, con la salvedad de que exista un consentimiento explícito del profesor, con la finalidad de que su uso no interfiera en el proceso de enseñanza-aprendizaje. Así, el alumno que no sigue el criterio establecido es penalizado, ya que falta a su responsabilidad profesional (cumplir la norma), y responsabilidad ética, ya que si el docente detecta como el alumno se distrae por el uso de los dispositivos electrónico y el conjunto de alumnos son los perjudicados, por la pérdida de tiempo productivo, y el posible efecto contagio (uso de dispositivos por parte de los alumnos de forma continua).

A partir de estos aspectos, esta comunicación evalua el uso no controlado que los alumnos hacen de los smartphones y otros dispositivos electrónicos en una asignatura de primer curso del Grado en Ingenieria Aeroespacial, así como se hace un análisis de los principales aspectos positivos y negativos de la implementación de estos dispositivos en la docencia.

\section{Objetivos}

El objetivo de este trabajo es analizar el comportamiento de los alumnos con respecto a la utilización de los dispositivos electrónicos a fin de establecer las medidas oportunas para que estos no interfieran en el proceso de enseñanza-aprendizaje durante las sesiones presenciales e incluso si es posible en un futuro implementar su uso en el proceso de docencia para potenciar el aprendizaje de los alumnos.

Concretamente, este trabajo se centra en evaluar el uso no controlado de las TIC's que hacen los alumnos, especialmente de los smartphones, para valorar el grado de adquisición de la responsabilidad ética y profesional, y al mismo tiempo, evaluar la posible implementación de los dispositivos electrónicos en la docencia.

\section{Análisis}

Para desarrollar nuestro objetivo se han llevado a cabo dos análisis.

En primer lugar, para conocer la situación de partida y poder evaluar el riesgo del uso no controlado de las TIC's, se realiza una encuesta al inicio del curso compuesta por 8 preguntas sobre los hábitos con las redes sociales y el smartphone en los alumnos.

La investigación se realiza sobre los estudiantes de primer curso del grupo de alto rendimiento académido (grupo ARA) de la asignatura "Empresa" impartida en el Grado en Ingeniería Aeroespacial de la Universidad Politécnica de Valencia en el curso 2018/2019. 
La muestra se compone de un total de 56 alumnos, 13 mujeres y 43 hombres. Todos ellos entre 17 y 18 años, y en su mayor parte procedentes de la ciudad de Valencia (España). Sin embargo uno de ellos no contestó ninguna de las preguntas del cuestionario, por lo que lo excluímos del análisis, reduciendo la muestra a 55 alumnos.

La encuesta realizada a los alumnos está estructurada de la siguiente manera:

Tabla 1. Modelo de cuestionario

1. Sexo:
a. $\mathrm{M}$
b. $\mathrm{F}$

2. Edad:

3. ¿Desde qué edad tienes smartphone?

4. ¿Consideras que el teléfono móvil es indispensable en tu vida?
a. Sí
b. No

5. ¿Qué redes sociales utilizas?
a. Washapp.
b. Instagram.
c. Youtube.
d. Twitter.
e. Otras.

6. ¿Cuánto tiempo has estado desconectado?
a. Nunca o rara vez.
b. Menos de 1 semana.
c. 1 semana.
d. Entre 2 y 3 semanas.
e. Más de 3 semanas.

7. ¿Te han llamado la atención alguna vez por usar el teléfono movil?
a. Sí
b. No.

8. ¿Quién usa con más frecuencia el telefóno movil?
a. Yo.
b. Mis padres.
c. Ambos.

Las dos primeras preguntas clasifican demográficamente a la muestra según su sexo y edad. Las preguntas 4 y 7 son de respuesta dicotómica, siendo 1 si la respuesta es afirmativa y 0 si es negativa. La pregunta 3 es abierta de respuesta numérica. Las preguntas 5 y 6 incialmente fueron inicialmente preguntas de respuesta abierta, y posteriormente a raíz de las respuestas obtenidas por los alumnos han sido codificadas a preguntas cerradas categóricas, resultando la pregunta 5 con 5 respuestas de opción múltiple, y la 6 de única respuesta también con 5 opciones. La pregunta 8 es de respuesta cerrada de única respuesta. 
En segundo lugar, para analizar la idoneidad del uso de los dispositivos electrónicos en el aula, se detalla el criterio actual seguido en la asignatura sobre su uso, acorde con la competencia transversal $\mathrm{N}^{\circ} 7$, y se estudia su implementación en la docencia de la asignatura mediante un análisis DAFO.

\section{Resultados}

4.1. Estudio inicial: Análisis sobre los hábitos de los alumnos respecto al uso del Smartphone.

Del análisis descriptivo y gráfico realizado sobre los cuestionarios se obtiene que los estudiantes obtienen su primer smartphone entre los 8 y los 16 años (figura 2), por lo que hay un amplio rango de edades en esta primera relación alumno-movil, sin embargo, la mayoría (un 70,9\%) de los estudiantes tuvo su primer smartphone entre los 12 y los 14 años, es decir, en los primeros años de la Educación Secundaria Obligatoria. También se observa que todos ellos acceden a la educación superior con smartphone propio.

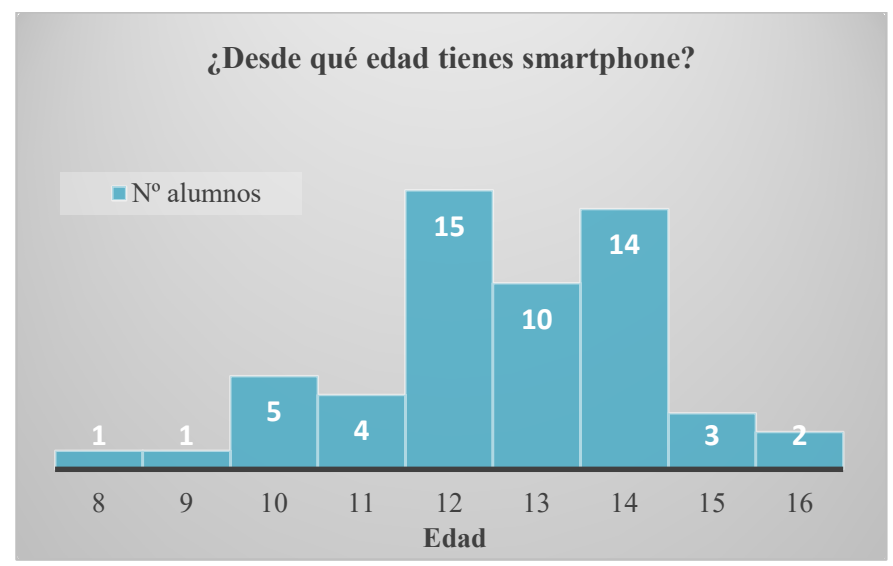

Fig.2 Análisis de la pregunta 3 del cuestionario.

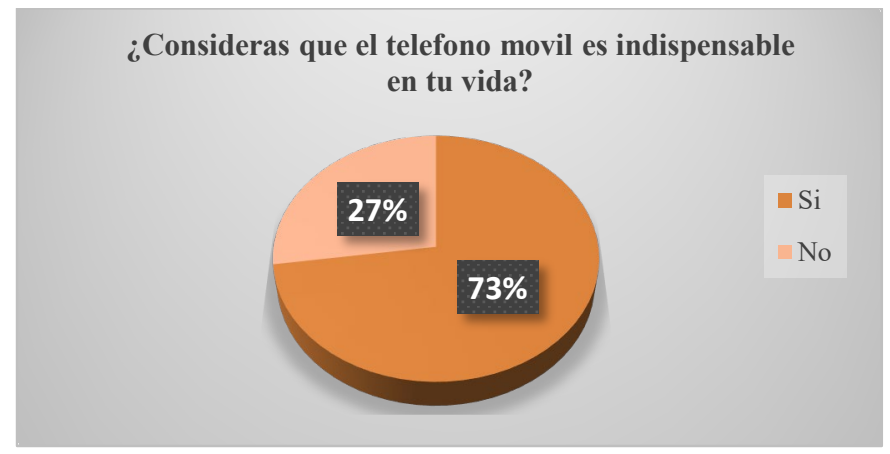

Fig. 3 Análisis de la pregunta 4 del cuestionario. 


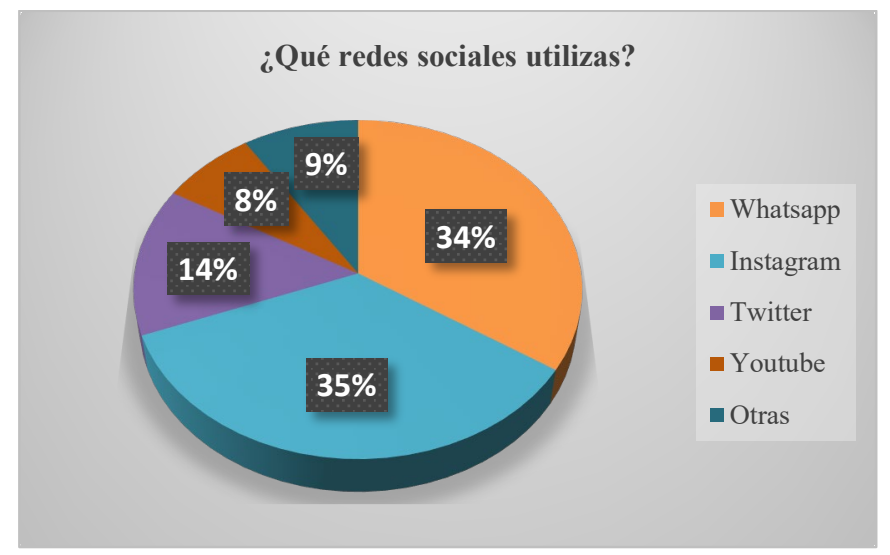

Fig. 4 Análisis de la pregunta 5 del cuestionario.

La figura 3 nos indica que el $73 \%$ de los alumnos considera que el teléfono movil es indispensable en su vida, aunque todavía hay un $27 \%$ de ellos que no se considera tan unido a su teléfono. Mientras que si analizamos las redes sociales más utilizadas, la figura 4 nos muestra que son Instagram, por un 35\% de los alumnos, y Whatsapp, con un 34\%. Con un uso más reducido le seguiría Twitter (14\%), Youtube (8\%), y otras redes $(9 \%)$, como Facebook, Pinterest, Snapchat, y Reddit.

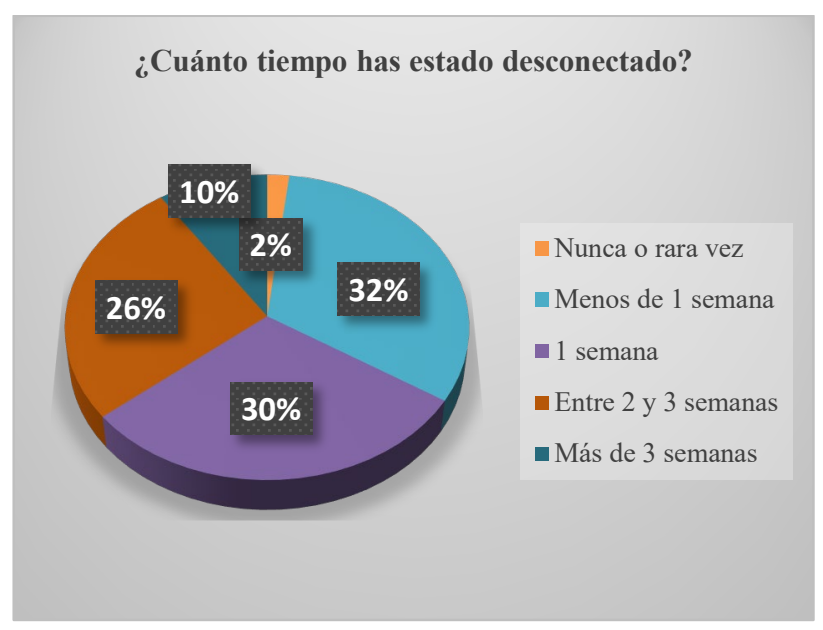

Fig. 5 Análisis de la pregunta 6 del cuestionario.

Sobre el tiempo que han permanecido desconectados de Internet a través de sus smartphones (figura 5) se observa que es bastante reducdio, el $32 \%$ solo ha estado desconectado unos pocos días (menos de 1 semana), el 30\% una semana exacta, y el 26\% entre 2 y 3 semanas. Tan sólo el $10 \%$ de los alumnos ha estado desconectado más de 3 semanas.

En la figura 6 se observa que existe bastante equilibrio entre los que sí que le han llamado la atención alguna vez por usar el móvil (47\%), y los que no (53\%) han sido regañados. 


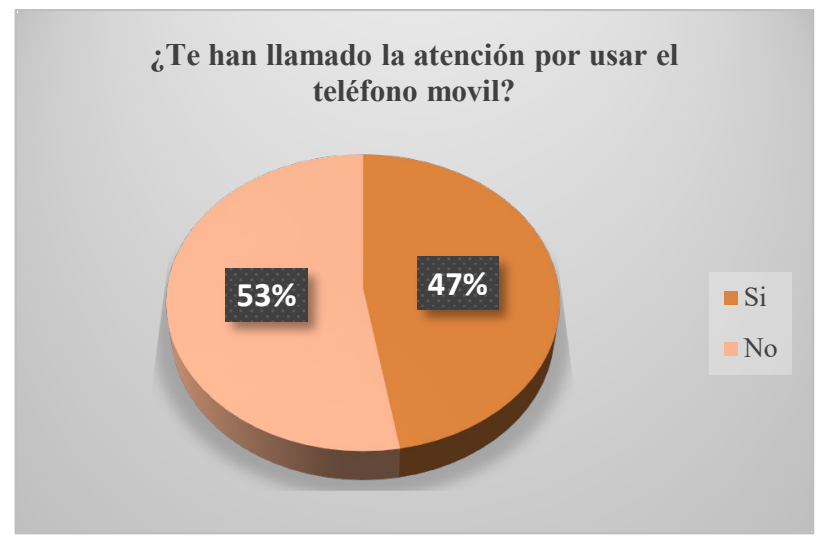

Fig. 6 Análisis de la pregunta 7 del cuestionario.

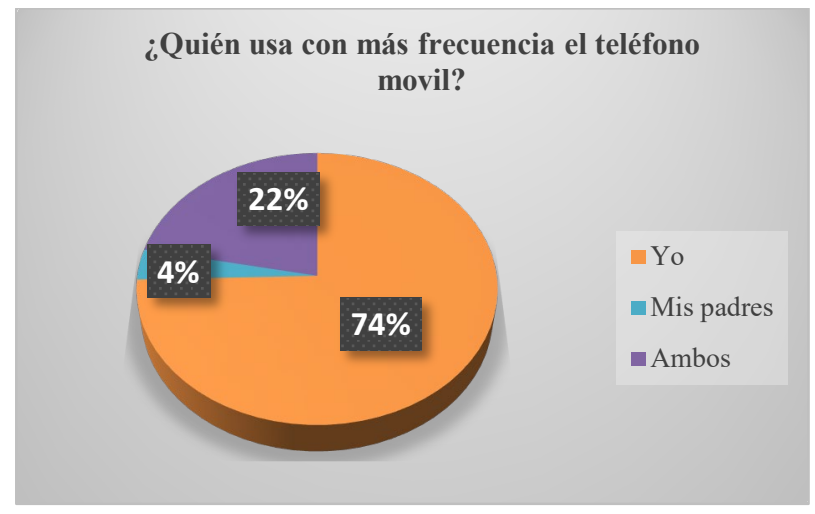

Fig. 7 Análisis de la pregunta 8 del cuestionario.

Por último, en la figura 7 se indica que el $74 \%$ de los alumnos considera que utiliza más el móvil que sus padres, aunque un $22 \%$ cree que la frecuencia de uso es la misma entre ambos. Sin embargo, tan sólo el $4 \%$ piensa que son sus padres quienes más utilizan el smartphone.

4.2. Desarrollo del criterio seguido en la asignatura "Empresa" sobre el uso de los dispostivos electrónicos y análisis DAFO sobre su implementación.

En la asignatura de "Empresa" impartida en el Grado de Ingeniería Aeroespacial de la UPV es punto de control la competencia transversal $\mathrm{N}^{\circ} 7$. Es por ello que, en relación a la responsabilidad ética y profesional, se evalúa la utilización de los dispositivos electrónicos en las sesiones de clase, concretamente el uso de ordenadores portátil, tablets y teléfonos móvil o smartphones. El criterio seguido en la asignatura es la prohibición del uso de estos dispositivos electrónicos en el aula, sin previo consentimiento del profesor, para no interferir en el proceso de enseñanza-aprendizaje. En el caso de no cumplir este criterio, el alumno es penalizado en el alcance de esta competencia.

Este criterio es evaluado mediante la observación a lo largo de las sesiones de clase. De esta manera, se conoce que de los 55 alumnos que componen nuestra muestra, 4 de ellos hicieron un uso no controlado y repetitivo de sus dispositivos electrónicos en el aula a lo largo del curso. Tras analizar las respuetas de estos 4 alumnos en los cuestionarios 
realizados al inicio del curso para identificar si tienen algún comportamiento en común, se detecta que las características comunes de estos 4 estudiantes son: todos ellos son varones, declaran utilizar con más frecuencia el móvil que sus padres, reconocen que han sido amonestados alguna vez, y todos adquirieron su primer teléfono móvil antes de los 13 años.

A pesar del criterio seguido en la asignatura sobre el uso de los dispositivos electrónicos, un uso adecuado de los mismos en la docencia también reporta beneficios al proceso de enseñanza-aprendizaje. Por ello, se estudian los principales aspectos que pueden llevar a la implementación de estos dispositivos en la docencia de la asignatura mediante un Análisis DAFO (tabla 2).

Tabla 2. Análisis DAFO sobre el uso de dispositivos electrónicos en la docencia.

\begin{tabular}{|c|c|}
\hline DEBILIDADES & AMENAZAS \\
\hline $\begin{array}{c}\text { - Interfiere en el proceso de enseñanza- } \\
\text { aprendizaje. } \\
\text { - Interfiere en la comunicación docente-alumno. } \\
\text { - Distracción o falta de atención respecto al } \\
\text { docente y a los contenidos de la asignatura. } \\
\text { - Disminución del rendimiento del alumno. } \\
\text { - El docente puede perder el control sobre el uso } \\
\text { adecuado o no que realice el alumno del } \\
\text { dispositivo movil. }\end{array}$ & $\begin{array}{c}\text { - Individuos adictos a los } \\
\text { dispositivos electrónicos. } \\
\text { - Falta de responsabilidad ética y } \\
\text { profesional derivada de la adicción } \\
\text { a los dispositivos electrónicos. } \\
\text { - Retroceso en valores como } \\
\text { consecuencia de la adicción (falta } \\
\text { de respeto). }\end{array}$ \\
\hline FORTALEZAS & OPORTUNIDADES \\
\hline $\begin{array}{l}\text { - Incentiva la participación en clase. } \\
\text { - Alumnos más motivados. } \\
\text { - Proceso de enseñanza-aprendizaje novedoso. } \\
\text { - Proceso de enseñanza-aprendizaje con un } \\
\text { enfoque más centrado en el alumno. } \\
\text { - El profesor puede utilizar aplicaciones móviles } \\
\text { que le faciliten las tareas docentes, como el } \\
\text { control de asistencia, la corrección de } \\
\text { ejercicios, o la asimilación de contenidos. } \\
\text { - Facilidad de asimilación de contenidos para el } \\
\text { alumno, ya que el proceso de enseñanza- } \\
\text { aprendizaje se puede basar en el juego. } \\
\text { - Acceso a materiales y recursos, más allá de los } \\
\text { que aporte el docente. }\end{array}$ & $\begin{array}{l}\text { - El uso respetuoso de los } \\
\text { dispositivos electrónicos deriva en } \\
\text { la adquisición de valores morales y } \\
\text { competencias, tales como la } \\
\text { responsabilidad ética y la } \\
\text { responsabilidad profesional. } \\
\text { - Enseñanza no presencial, sin } \\
\text { dependencia del tiempo ni del } \\
\text { espacio. }\end{array}$ \\
\hline
\end{tabular}

Fuente: Elaboración propia. 


\section{Conclusiones}

Con el uso de las redes sociales se favorece el desarrollo de diferentes competencias: comunicativa, lingüística, digital, tratamiento de la información, aprender a aprender, autonomía y iniciativa personal (Martí y Garcia, 2014).

Sin embargo, tan importante es desarrollor e implentar su uso en la educación, como el hecho de saber controlarlo en determinados momentos de las fases de aprendizaje. De hecho, el buen uso de las tecnologías de la información, especialmente de los dispositivos electrónicos móviles, también supone la adquisión de determinadas completencias clave en el desarrollo integral del alumno, concretamente la adquisición de la responsabilidad ética y profesional.

En este estudio se ha evaluado, en primer lugar, el grado de uso de las redes sociales y los smartphones manifestado por los propios alumnos de la asignatura Empresa impartida en primer curso de Ingeniería Aeroespacial, para conocer si se les puede considerar capaces de ser responsables en el uso de estas herramientas.

El 73\% de los alumnos considera que el teléfono movil es indispensable en su vida, siendo entre 12 y 14 años la edad en que lo adquirieron por primera vez en el 70,9\% de los casos, mientras que todos ellos acceden a la educación superior con smartphone propio. Tambíén hemos observado que tan sólo un $10 \%$ de los alumos ha estado desconectado de Internet a través de su smartphone un período relativamente largo (más de 3 semanas), y que el 74\% considera que utiliza el móvil más que sus padres. Respecto al uso de las redes sociales, las más utilizadas son Instagram (35\% de los alumnos) y Whatsapp (34\%). Sin embargo, por otra parte, se observa que a un 53\% nunca le han llamado la atención por usar el móvil, mientras que a un $47 \%$ sí que lo han hecho alguna vez.

Con este análisis observamos que hoy en día, los alumnos acceden a la educación superior con unos hábitos ya creados acerca del uso del móvil y con un elevado nivel de vinculación con sus smartphones, por lo que se pone de manifiesto la necesidad de trabajar la responsabilidad ética y profesional derivada del uso de esta herramienta en las etapas educativas anteriores, pero sin descuidarla en la etapa universitaria, ya que es el último escalón antes de acceder al mundo profesional.

En segundo lugar, se ha revelado el criterio seguido en la asignatura a lo largo del curso sobre el uso de los dispositivos electrónicos, ya que no se permite su uso para no interferir en el proceso de enseñanza-aprendizaje. Sin embargo, los docentes de la asignatura son conscientes de los aspectos positivos que también puede reportar su implementación en el aula, es por ello que se ha estudiado dicha implementación a través de un análisis DAFO. En el análisis se observa que la integración de los dispositivos electrónicos en la docencia cuenta con un conjunto variado de aspectos positivos y negativos, con lo que resulta complicado decidir sobre si es o no apropiada su implementación en el aula. En cualquier caso, antes de tomar una decisión, el docente deberá corregir o mitigar las debilidades, y también ser consciente de las amenazas, así como capaz de afrontarlas. Los principales aspectos negativos de su implementación se derivan de la posible perdida de control por parte del docente para saber si el alumno está realizando en todo momento un uso adecuado 
del dispositivo, y la distracción por parte del alumno. Aunque es difícil asegurar la completa corrección de estos inconvenientes, el docente debe ser consciente que el implementar estas herramientas en la docencia, también reporta importantes beneficios al proceso de enseñanza-aprendizaje, por lo que debe apoyarse en ellos para mantener las fortalezas y explotar las oportunidades que se desarrollen de su integración en el aula.

Consideramos importante favorecer la difusión de este tipo de trabajos que pone en evidencia la realidad, captada de primera mano, sobre el uso de los dispositivos electrónicos, en especial de los smartphones, con el fin de contribuir a aumentar la consciencia sobre su uso entre el propio alumnado, y de esta manera favorecer el desarrollo de sus competencias éticas y profesionales tanto en el entorno educativo como fuera del mismo. Además, es evidente, que la integración de los dispositivos electrónicos en la docencia va a crecer en gran medida en los próximos años, por lo que este trabajo contribuye a su implementación en la educación superior universitaria.

\section{Referencias}

ALONSO MOSQUERA, M.H, GONZÁLVEZ VALLÉS, J.E, y MUÑOZ DE LUNA, A.B. (2016). "Ventajas e inconvenientes del uso de dispositivos electrónicos en el aula: Percepción de los estudiantes de Grados en Comunicación”. Revista de Comunicación de la SEECI, 41, 136-154.

ARNÁIZ UZQUIZA, V., y ÁLVAREZ ÁLVAREZ, S. (2016). "El uso de dispositivos y aplicaciones móviles en el aula de traducción: Perspectiva de los estudiantes". Revista Tradumàtica: Tecnologies de la Traducció, 14, 100-112.

BRAZUELO GRUND, F., GALLEGO GIL, D.J., y CACHEIRO GONZÁLEZ, M.L. (2017). "Los docentes ante la integración educativa del teléfono móvil en el aula". Revista de Educación a Distancia, 52, 6.

DOMINIC CHAWINGA, D. (2017). "Taking social media to a university classroom: teaching and learning using Twitter and blogs". International Journal of Educational Technology in Higher Education, 14, 3.

HERNÁNDEZ, J. (2010). "La ética profesional, ¿un problema ético del contador público?”. En Revista del Centro de Investigación de Ciencias Administrativas y Gerenciales, vol. 7, $\mathrm{N}^{\circ}$ 1, p. 73-88.

IBARRA ROSALES, G. (2005). "Ética y formación profesional integral”. En: Reencuentro, vol. 43, p. 1-13.

MARTÍ CLIMENT, A. y GARCIA VIDAL, P. (2014). "El treball per projectes amb les TIC a l'àrea de Llengua $i$ Literatura. Una experiència didàctica en la formació del professorat de Secundària”. Articles de Didàctica de la Llengua i la literatura, 64, 1-10.

MARTÍ CLIMENT, A. y GARCIA VIDAL, P. (2018). "Redes sociales en la enseñanza superior". En Congreso Nacional de Innovación Educativa y Docencia en Red (IN-RED 2018). Valencia, Spain: Editorial UPV. Páginas 141 - 154.

MESO AYERDI K., PÉREZ DASILVA J.Y., y MENDIGUREN GALDOSPIN, T. (2011). "La implementación de las redes sociales en la enseñanza superior universitaria". Tejuelo, $12,137-155$. 
El uso de los dispositivos electrónicos móviles como herramienta docente de una asignatura de Grado

NAVÉS, F.A. (2015). "Las TIC como recurso didáctico: ¿Competencias o posición subjetiva?". En Revista de Investigación Educativa 20, 238-248.

ORGANISTA SANDOVAL, J., y SERRANO SANTOYO, A. (2011). "Implementación de una actividad educativa en modo colaborativo con apoyo de smartphones: Una experiencia universitaria". Revista Electrónica de Tecnología Educativa, 36.

TAPSCOTT, D. (1998). Creciendo en un entorno digital: la Generación Net. New York: McGraw-Hill.

UPV (2019). La responsabilidad ética, medioambiental y profesional como competencia transversal. $\quad<$ http://www.upv.es/contenidos/COMPTRAN/info/954882normalc.html>

[Consutado: 8 de febrero de 2019] 